\title{
PERIPHERAL MEMBERS OF M67
}

I. PLATAIS AND V. KOZHURINA-PLATAIS

Yale University Observatory

P.O. Box 208101, New Haven, CT 06520, U.S.A.

M. GEFFERT

Sternwarte der Universität Bonn

Auf dem Hügel 71, D-5300 Bonn, Germany

AND

V. N. FROLOV

Pulkovo Observatory

196140 St.-Petersburg, Russia

Despite enormous effort put into the membership determination of M67, we do not know very well its peripheral members. Since the Bonn double astrograph as well as Pulkovo normal astrograph plates cover nearly $1: 5 \times 1.5$ on the sky we have combined newly derived astrometric data from both instruments. Nearly 2,000 stars were measured down to the plate limit $B \approx 16.5$. The proper-motion standard error for well-measured stars is $\pm 0.3 \mathrm{mas} / \mathrm{yr}$. For the membership probability solution we selected only 1363 stars, all brighter than $V \approx 15.0$, with good proper motions. The membership solution yielded 446 cluster members showing convincing discrimination between the cluster and field stars. The spatial distribution of peripheral cluster members seems to be asymmetric, although, it does not indicate that the tidal radius of the cluster has been reached.

Summarizing, we believe that with additional photoelectric photometry and radial velocity measurements M67 could become a true "standard candle" for the studies of solar-type and age stars. 